\title{
Values and Disadvantages of Outsourcing the Regulatory Affairs Tasks in the Pharmaceutical Industry in EU Countries
}

Gummerus $A^{1^{*}}$, Airaksinen $\mathbf{M}^{2}$, Bengtström $\mathbf{M}^{3}$ and Juppo $A^{3}$

${ }^{1}$ Oy Medfiles Ltd., Hatanpään valtatie 26 A, 33100 Tampere, Finland

${ }^{2}$ Department of Pharmacy, University of Helsinki, Finland

${ }^{3}$ Department of Pharmacy, Abo Academy University, Finland

*Corresponding author: Gummerus A, Oy Medfiles Ltd., Hatanpään valtatie 26 A, 33100 Tampere, Finland, Tel: +358 407237031 ; E-mail: anu.gummerus@medfiles.eu

Received date: March 14, 2016; Accepted date: April 12, 2016; Published date: April 15, 2016

Copyright: ( $) 2016$ Gummerus A, et al. This is an open-access article distributed under the terms of the Creative Commons Attribution License, which permits unrestricted use, distribution, and reproduction in any medium, provided the original author and source are credited.

\begin{abstract}
Background: The purpose of this study was to determine the values and disadvantages of outsourcing regulatory affairs tasks in the pharmaceutical industry. The aim was also to study how many CROs the pharmaceutical companies outsource regulatory affairs tasks to and the duration of outsourcing partnerships between companies and CROs.
\end{abstract}

Method: The study was conducted as an e-mail survey in the pharmaceutical industry in Finland, Sweden, Estonia, Germany and Spain focusing on those companies that undertake regulatory affairs.

Results: The survey received 71 completed responses out of 147 , a response rate of $48 \%$. According to the responses, $65 \%$ of the pharmaceutical companies have outsourced tasks related to research and development over the last three to five years. Over $44 \%$ of the respondents informed that they have outsourced to one or two CROs only. One quarter of the respondents have outsourced to three to five CROs. Most (91\%) of the respondents in the pharmaceutical industry strongly agree and agree on the fact that they outsource the regulatory affairs tasks because they want to obtain greater flexibility. The companies evaluated that outsourcing to CROs is expensive (strongly agree or agree $74 \%$ ).

Conclusion: CROs have to keep the qualitative level high and obtain flexibility towards the companies. When a company is considering outsourcing regulatory tasks, planning has to be done well in advance. The main topics to be discussed between the company and CRO before outsourcing process are the estimated costs of the outsourcing, outsourcing strategy, information flow and audit trails.

Keywords: CRO; Regulatory affairs; Outsourcing; Pharmaceutical industry

\section{Introduction}

Since the 1980s, the pharmaceutical industry has experienced a change in the market situation, involving mergers, acquisitions, internationalization, consolidation, downsizing and changing regulatory trends. Due to this evolution, companies have had to reallocate funds and resources to research and development. As a consequence, the pharmaceutical industry has increasingly started to rely on partnership relations with outside Contract Research Organizations (CRO) [1,2]. Companies use outsourcing to enter the market in order to avoid time delays in hiring and infrastructure development as well as to prevent internal resistance to new ideas. A pressing question for pharmaceutical companies is how to best organize the $\mathrm{R} \& \mathrm{D}$ activities in order to improve productivity and flexibility: Which activities to keep in-house and which to outsource to CROs? Avellanet [3] reports on a U.S. survey of over 13,000 executives from medical device and biopharmaceutical industries that single-task focused roles within regulatory affairs and quality departments are being increasingly outsourced. Quality agreements between a CROs and companies are becoming change management control agreements. The reliability and quality of services, including the economic stability of the CRO, are assessed through a lengthy process involving on-site audits. Many pharmaceutical companies already work together with multiple CROs during the entire innovation process.

The outsourcing of the regulatory affairs function has been a growing trend, yet it is a topic that has been subject to very little discussion [4]. The regulatory affairs and operations processes that are outsourced involve medical writing, submission planning and publishing, regulatory data and information management, local regulatory affairs, pharmaceutical-chemical writing, labeling, agency liaison, regulatory strategy, translation, administrative documents, dossier conversion and literature searches [5]. According to Avallanet [3] the regulatory affairs functions most likely to be outsourced include labeling, CTD/eCTD assembly, training and submission tracking, indexing and archival. The role of a regulatory affairs expert is not restricted to the registration of products; they advise companies both strategically and technically. Their role begins right from the development of a product to manufacturing, marketing and postmarketing strategies. Nowadays regulatory affairs experts require answers to the question of "how" from a strategic point of view. The regulatory affairs expert must balance four concerns: patient safety, 
product efficacy, regulatory compliance and company profitability. The regulatory affairs expert should therefore be a member of the team deciding the regulatory and launch strategy [6].

As stated by Teirlinck and Spithoven [7] and Ranki-Pesonen and Soppi [8] companies wish to restrict the number of CROs to collaborate with. This signifies that the CROs they select tend to be rather larger than smaller in size and are therefore able to provide an array of services. Working more closely with fewer providers in theory makes the process more efficient by simplifying the outsourcing network and improving collaboration between CROs and companies. Whereas the largest CROs are often an automatic choice for major projects, pharmaceutical companies are increasingly turning to medium-sized CROs, which can be more responsive and flexible. These CROs can retain a more personalized service for a company [1].

According to Getz [9], Piachaud [10,11] and Vogel and Getz [12] a number of benefits could be accrued from outsourcing manufacturing processes to CROs in the pharmaceutical industry. Outsourcing to CROs enables companies to buy specialized knowledge and skills, as well as to facilitate a rapid exploitation of technology. Likewise, this gives companies the freedom to concentrate on their core functions [9-12]. Additionally, the companies can spread out risks by outsourcing various tasks to CROs [13].

Based on the literature review it was revealed that even if tasks related to product development are outsourced in the pharmaceutical industry, the outsourcing tasks and processes of regulatory affairs have not been widely studied. Nonetheless, regulatory affairs do have an important role in product development and maintenance strategy along with business plans [14].

The purpose of this study was to determine the values and disadvantages of outsourcing regulatory affairs tasks in the pharmaceutical industry. The aim was also to study how many CROs pharmaceutical companies outsource regulatory affairs tasks to and the duration of outsourcing partnerships between companies and CROs. This paper is the second part of a survey done in the EU. The first part of the survey covered the questions why and what regulatory affairs tasks are being outsourced [15].

\section{Methods}

The study was performed as an email survey in the pharmaceutical industry in Finland, Sweden, Estonia, Germany and Spain, focusing on those companies that undertake regulatory affairs. The countries concerned were selected in order to represent different European Union countries: Finland and Sweden are Nordic countries; Estonia joined the EU in 2004, while Germany and Spain are big economies in the EU. There are also cultural differences between the north and south part of the EU, which gives greater variety to the countries chosen for this study.

The survey was designed to cover the main questions relating to the present outsourcing situation, and the reasons, values, disadvantages and future of outsourcing of regulatory affairs. Some of the questions were emphasized by Piachaud's study of outsourcing in the pharmaceutical manufacturing process, which was performed in 2002 [10]. Piachaud's study was conducted among the pharmaceutical industry and some of the questions were also appropriate for this study. The survey consisted of structured questions, and, where applicable, an open field was included to allow the respondents to indicate issues that were not included in the structured question. In addition, space was made available for open responses/comments at the end of the survey. Some of the questions were designed so that the respondent was able to select multiple options. Anonymity was required due the confidentiality issues relating to the pharmaceutical industry. Therefore, the anonymity of the respondents was also assured in this questionnaire. For quantitative analysis of the responses, multiple-choice responses were categorized on a five-point Likert scale. A "no-opinion" option was added to ensure that "neutral" was not selected for "no opinion" answers. These "no opinion" answers were not taken to account when calculating the average value.

To assure face validity of the questionnaire, a pilot survey was conducted in late 2010. The pilot survey was sent to five academic and/or industrial experts. The responses of the pilot study were not included in the results of the final study. Two comments were received concerning the linguistic form of the questionnaire. After making the suggested changes to the content of the questionnaire, the main phase on the survey started in March 2011 and ended in August 2011. The list of responding pharmaceutical companies was provided by Pharma Industry Finland. The corresponding organizations from Sweden, Estonia, Spain and Germany provided their regulatory affairs contact list. Generic Medicines Association Finland also provided their regulatory affairs contact list. The companies represented all sorts of companies: innovation based and generic companies, large, international companies, subsidiaries, CROs, CMOs and small/ medium-sized stand-alone companies. If the regulatory affairs director or manager or a person of similar authority was not known, the questionnaire was sent to the quality or clinical manager or a person of similar authority. The questionnaire was sent to one person in each company. The responding to the survey was done electronically via Helsinki University E-survey tool. The results were analyzed with SPSS and transferred to Excel software for quantitative analysis. The results are presented as percentages and frequencies. Responses to the openended questions were content analyzed by grouping similar themes and ideas [16]. Some representative responses are referred to in the results section.

\section{Results and Discussion}

In total, the survey received 71 completed responses out of 147 questionnaires sent, giving a response rate of $48 \%$. The majority (59 out of 147) of responses were from Finland and Sweden (Table 1).

\begin{tabular}{|c|c|c|c|}
\hline Country & $\begin{array}{c}\text { Number of sent } \\
\text { questionnaires (n) }\end{array}$ & $\begin{array}{c}\text { Responses } \\
\text { received (n) }\end{array}$ & $\begin{array}{c}\text { Response rate } \\
\text { (\%) }\end{array}$ \\
\hline Finland & 46 & 29 & 63 \\
\hline Sweden & 64 & 30 & 47 \\
\hline Estonia & 22 & 3 & 9 \\
\hline Spain & 6 & 4 & 55 \\
\hline Germany & 9 & 5 & 48 \\
\hline Total & 147 & 71 & 67 \\
\hline
\end{tabular}

Table 1: Location of the pharmaceutical companies responding to the survey $(n=71)$.

The response rate in Estonia was poor, 9\%, even though the questionnaire was sent three times. When the results were studied closely, the responses did not separate if the respondent was from 
Finland, Sweden or other countries. For this reason, results from different countries were combined.

Most of the respondents (80\%) worked in a subsidiary of a worldwide, international company. This reflects the fact that there are not many headquarters located in the countries involved in this survey. Moreover, $64 \%$ of the companies that responded had proprietary products for human use ( $85 \%$ human medicines). Many of the respondent companies also dealt with medical devices (48\%).

Most commonly the responder was a person with the title "head of the regulatory affairs" (62\% of the respondents), but there were also several other titles, such as regulatory affairs manager or similar $(22 \%)$, head of the company (5\%) and other titles (10\%), such as head of quality assurance, senior regulatory manager, responsible pharmacist, vice president or clinical development and regional regulatory affairs head. The titles depend on the organizational structure of the company. Even so, the questionnaire reached well the persons in charge of the company's regulatory affairs functions.

According to the responses received, $65 \%$ of the pharmaceutical companies have outsourced tasks related to research and development over the last three to five years (Figure 1).

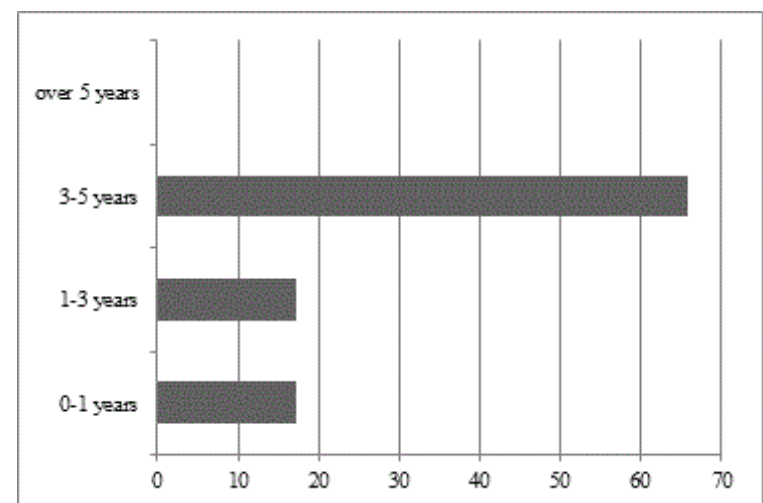

Figure 1: Number of years (\% of the respondents) the companies have outsourced regulatory affairs tasks.

None of the respondents in this survey had outsourced for more than five years. The most outsourced tasks were regulatory affairs ( $72 \%$ of the respondents) and clinical trials (55\% of the respondents). Outsourcing of regulatory affairs tasks as an operation procedure was familiar to the companies. This survey indicates that outsourcing regulatory affairs has become a required procedure during the last decade. In the same way, tasks concerning clinical trials, production, quality assurance and pharmacovigilance have been outsourced, but these tasks were not defined more precisely in the survey and are not part of this paper.

Strategies and deal structures differ from company to company, but many of the leading pharmaceutical companies have made efforts to work with fewer CROs in late-stage development. Working more closely with fewer providers in theory makes the process more efficient by simplifying the outsourcing network and improving collaboration between CROs and companies [17]. It seems that companies prefer to use the same CROs (Figure 2). Over 44\% of the respondents informed that they have only outsourced to one or two CROs. One quarter of the respondents have outsourced to three to five CROs.

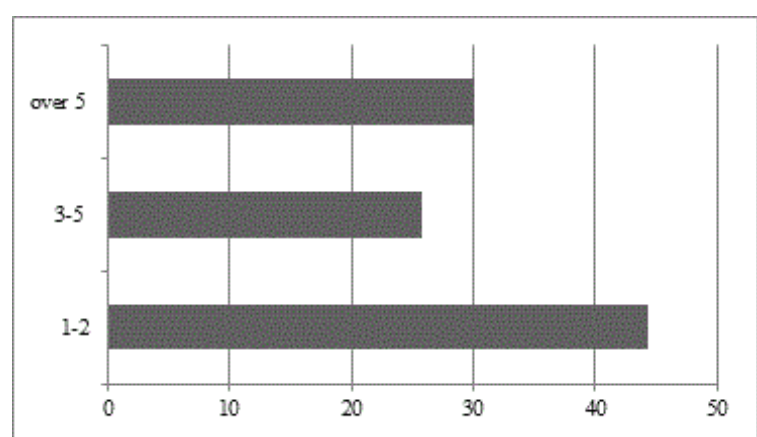

Figure 2: The number of CROs the companies (\% of the respondents) outsourced to three to five CROs.

One response to the open-ended question stated: "no outsourcing to different CROs due the fact that the quality of service deteriorated". Responses from another point of view were also received. One respondent expressed that "the companies do have contacts to several CROs in order to qualify. Example, ten text translations within three days." Depending on the urgency of the work task, the number of CROs used may vary. If the company has many CROs, coordination work between the company and the CRO contact persons will increase. Big pharmaceutical companies need outsourcing to CROs in situations involving a lack of resources, whereas smaller companies are dependent on overall specialty know-how CRO that can provide an addition to the acquired specific service.

\section{The values of outsourcing regulatory affairs tasks}

Most (91\%) of the respondents in the pharmaceutical industry either agree or strongly agree on the fact that they outsource regulatory affairs tasks because they want to obtain greater flexibility (Figure 3).

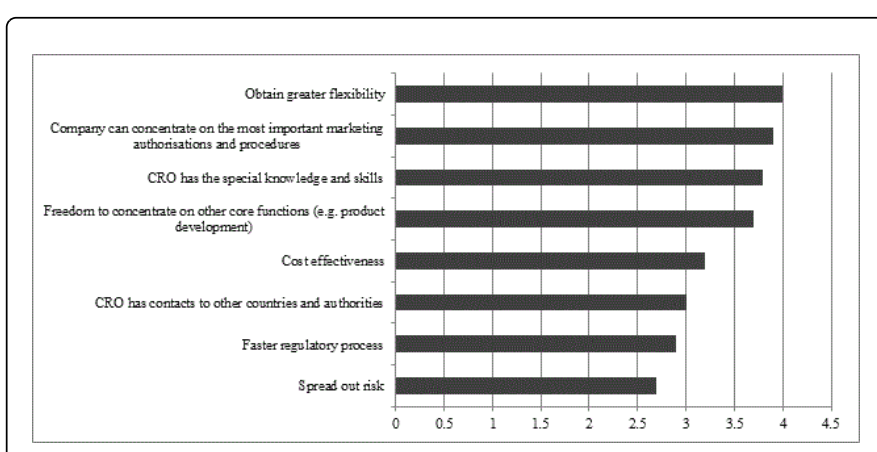

Figure 3: Values of outsourcing regulatory affairs $(1=$ strongly disagree, 2 = disagree, 3 = neither agree nor disagree, $4=$ agree, $5=$ strongly agree).

Flexibility was especially highlighted in the replies to the openended questions. One respondent pointed out that a "CRO is used to handle temporary high workload." In practice this means that a CRO can provide quick assistance in a task that is urgent, that can be outsourced on project basis or that would otherwise burden the company's regulatory affairs personnel.

When pharmaceutical companies outsource, it is important that the CROs can perform pre-defined tasks. According to the survey, $70 \%$ of 
the respondents in the pharmaceutical industry outsource as CROs have special knowledge and skills. A common situation can be that the pharmaceutical company does not have sufficient knowledge of different marketing authorization procedures. One respondent wrote: "Especially expert help in country specific issues is required". The CRO network was not highly acknowledged; only $11 \%$ strongly agreed that they value this network covering other countries and authorities. Many big pharmaceutical companies have their own subsidiaries in the countries they wish to contact and therefore the CRO network is not a requirement.

A major advantage of outsourcing is that companies can concentrate on their most important marketing authorizations and procedures (69\%), in addition to other core functions (such as product development processes). Similarly, the companies can focus on their top priority procedures and launch products by the deadline. This means that the tasks transferred to CROs are not top priority procedures. The tasks may range from variations to translations [16].

Many companies in the pharmaceutical industry would like to have their products approved as soon as possible to launch them to the market. However, in the results of this survey, faster regulatory processes done by CROs were not highlighted as having worthwhile advantage. One third of the respondents agreed or strongly agreed with the claim. This result may reflect the fact that companies want to concentrate on their most important marketing authorization procedures. In conclusion, it was not seen as important that a CRO is hired to do faster regulatory processes as companies execute the most significant processes themselves.

Contrary to what was expected, cost-effectiveness was not one of the top values of the respondents in this survey. Moreover, only 7\% of the respondents strongly agreed and 35\% agreed that they see reduced costs valuable in outsourcing. This might result from the fact that the respondents were not necessarily in the position to evaluate the cost of outsourcing regulatory affairs. According to the literature review, costeffectiveness is one of the main reasons for companies to undertake $R \& D$ outsourcing $[10,17,18]$. The outsourcing literature recognizes that many organizations fail to realize the financial benefits sought by outsourcing. Further, prior research has found that outsourcing organizations struggle to estimate accurately the so-called "hidden costs" associated with managing these inter-organizational relationships. This is especially true of complex, globally distributed outsourced services. According to the literature, the scale and geographic distance of the outsourcing service increase both control and coordination costs. The breadth of tasks and geographic dispersion are found to lead to greater control costs but not coordination costs $[19,20]$. According to Piachaud [11], outsourcing can make a significant contribution toward reducing the firm's overall cost base.

According to the responses, spreading out risks was not seen as an important value of outsourcing regulatory affairs. Outsourcing to a few or several CROs may spread out risks. The risks were not defined more closely in the study. Furthermore, CRO quality can be controlled by comparing the efficacy, speed and quality of work in different CROs. To avoid unwanted consequences, all risks should be evaluated, analyzed and minimized, and a risk management plan should be developed.

\section{The disadvantages of outsourcing regulatory affairs tasks}

The companies evaluated that outsourcing to CROs is expensive (strongly agree or agree $74 \%$ ) (Figure 4).

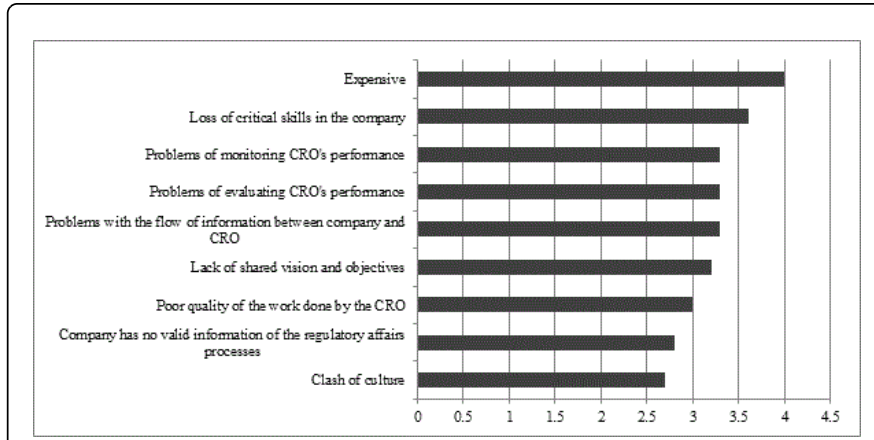

Figure 4: Disadvantages of outsourcing regulatory affairs $(1=$ strongly disagree, $2=$ disagree, $3=$ neither agree nor disagree, $4=$ agree, 5 = strongly agree).

Nevertheless, the companies attempt to gain financial benefit from outsourcing. The survey did not evaluate if the assumption was based only on the respondent's instinctual opinion or on a profit and loss account. Firstly, the respondents were not necessarily in the position to evaluate the cost of outsourcing regulatory affairs. Secondly, when outsourcing is being discussed about in a company, the possible consequences should be evaluated carefully in a risk management plan of outsourcing; what are the costs if the outsourcing of regulatory affairs is discontinued. There is always the possibility that the cooperation does not work. Even so, switching CROs is also expensive. Also, the outsourcing policy may be changed in a way that the company back sources the regulatory affairs tasks in-house. According to Whitten et al. [21], outsourcing continuation is the most preferred and back sourcing the least preferred option when switching costs are high, and that the relative preference for CRO switching depends on the switching cost type.

The companies also dread that if they outsource regulatory affairs tasks to CROs, they will lose critical skills in the company. Half of the respondents strongly agreed or agreed with this claim. On the other hand, $70 \%$ of the respondents think that CROs have special knowledge and expertise to perform regulatory affairs tasks. When a company outsources regulatory affairs tasks, it has to plan carefully what tasks are to be outsourced to avoid losing critical regulatory affairs skills in the company. If the company does not have expertise in that special field, it might be more cost-effective and qualitative to outsource these tasks to CROs. As mentioned previously, the company must plan the outsourcing to avoid extra work inside the company. One respondent commented: "It is hard to find a CRO that would take full regulatory responsibility of a product". Another respondent remarked: "Sometimes it is easier to do it yourself than explain." It certainly is a challenge for CROs to get knowledge and updated information on a product or process when it is outsourced. When a work task is transferred to a CRO, it is the responsibility of a professional CRO to ask for specific details related to the process and/or product in question. It is essential that CROs have enough resources to obtain flexibility in view of the fact that when outsourced regulatory expertise is required, the situation in a company may be urgent. CROs must also have resources for situations of this kind.

The respondents were asked how they experience the relationship between a CRO and the outsourcing company. Approximately half (52\%) of the respondents confirmed that they have had problems with the flow of information between the company and a CRO. It was not 
specified if there had been a contact person on both sides or not Nevertheless, the information flow between a CRO and the company was regarded as challenging. The respondents were also asked how they evaluate and monitor the performance of a CRO. As in any business, the company should have the opportunity to check and audit the CRO's quality of work. Almost half of the respondents recognized that they have experienced problems. However, the respondents disagreed or strongly disagreed with the claim of poor quality of work done by CROs. This means that the respondents were satisfied with the quality of work, but had had problems to evaluate and monitor the performance. According to one open-ended response, "The service providers do not always follow the given procedure." This is a very interesting claim that should be studied more closely to ascertain if CROs have their own procedures which they follow instead and despite of the clients' requests. In the initiation of the outsourcing process, standard operation procedures and work instructions should be defined and given to the persons concerned.

The respondents evaluated that a clash of culture was not an issue when outsourcing regulatory affairs. Only $14 \%$ of the respondents strongly agreed or agreed with this claim. For organizations to work together, it is imperative that there still be some degree of cultural fit and compatibility between them. Different enterprise or social cultures may cause the clash of culture. In these cases, senior management must take an active role in the outsourcing process. Although there is a view that by building longer-term relationships pharmaceutical companies will get the best out of their CRO partners, a successful relationship inevitably implies that efforts are made by both parties if a successful outcome is to be ensured. Likewise, a practical communication plan between the company and the CRO has to be developed, managed and kept updated. The value of knowing the individual contact is very important $[22,23]$. In order for a company to be successful in the management of the outsourcing process, an internal policy should be developed. The implementation of outsourcing activities within a company should be performed in an efficient and effective manner. In many occasions, outsourcing is performed on an individual basis which leads to many concerns, such as higher costs, poor responsiveness, and lack of partnership relations, shared visions and objectives. The development of an outsourcing process would provide the most efficient and effective approach to outsourcing [24].

\section{Conclusion}

The main conclusion of this survey is that CROs have to keep the qualitative level high and obtain flexibility towards the companies. When a company is considering outsourcing regulatory tasks, planning has to be done well in advance. Based on this study, the main topics to be discussed between the company and CRO before outsourcing process are the estimated costs of the outsourcing, outsourcing strategy, information flow and audit trails. If outsourcing becomes necessary due a lack of resources, the amount of work to be outsourced needs to be defined. This simplifies the outsourcing process to the CRO. The CRO benefits from this information and is able to plan their resources and provide more flexibility. The outsourcing strategy of the company has to also be informed to the appropriate person internally in the company so that all parties are aware of the company's outsourcing policy.

Based on this study, the pharmaceutical industry outsource as CROs have special knowledge and skills but on the other hand, the companies also dread that if they outsource regulatory affairs tasks to CROs, they will lose critical skills in the company. Regular and honest communication is vital in order to achieve a good relationship.

\section{References}

1. Hassanzadeh F, Modarres M, Nemati HR, Amoako-Gyampah K (2014) A robust $R \& D$ project portfolio optimization model for pharmaceutical contract research organizations. Int. J. Production Economics 158: 18-27.

2. Mitchell E (1997) Trends in outsourcing drug development and manufacturing. Pharmaceutical technology 21: 64-68.

3. Avellanet J (2010) Get to market now! Turn FDA compliance into a competitive edge in the era of personalized medicine, Logos Press.

4. Leduc CE, Mantus DS (2000) Outsourcing regulatory affairs in the biotechnology and life science sectors. J of Biolaw and Business 4: 28-39.

5. KPMG Outsourcing in the pharmaceutical industry: 2011 and beyond.

6. Kennedy T (2001) Strategic project management at the project level. Clin Research and Reg Affairs 18: 345-365.

7. Teirlinck P, Spithoven A (2013) Research collaboration and R\&D outsourcing: Different R\&D personnel requirements in SMEs. Technovation 33: 142-153.

8. Ranki-Pesonen M, Soppi E (2012) Survey of Finnish vendors serving the pharmaceutical industry: culminatum innovation.

9. Getz K (1997) The expanding outside clinical services contractor marketplace. Clinical Research and Regulatory Affairs 14: 191-204.

10. Piachaud B (2002) Outsourcing in the pharmaceutical manufacturing process: an examination of the CRO experience. Technovation 22: 81-90.

11. Piachaud B (2005) Outsourcing technology. Research Technology Management May/June 48: 40-46.

12. Vogel JR, Getz KA (1997) Factors driving the increased use of outside contractors in drug development. Clinical Research and Regulatory Affairs: $177-190$

13. Quinn J (2000) Outsourcing innovation: the new engine of growth. Sloan management review 41: 4 .

14. Kumar M, Tate K (2008) Designing a global product development strategy. Global harmonization: $1-19$.

15. Gummerus A, Airaksinen M, Bengtström M, Juppo A (2016) Outsourcing of regulatory affairs tasks in pharmaceutical companies why and what? J Pharm Innov 11: 46-52.

16. Patton MQ (1990) Qualitative evaluation and research methods (2nd edn.) Newbury Park: Sage Publications.

17. Howells J, Gagliardi D, Malik K (2008) The growth and management of R\&D outsourcing: evidence from UK pharmaceuticals R\&D Management 38: 205-219.

18. Caudy DW (2001) Using R\&D outsourcing as a competitive tool. Medical Device and Diagnostic Industry Magazine, MMDI Archive.

19. Kumar S, Snavely T (2004) Outsourcing and strategic alliances for product development: a case of Banta Digital Group. Technovation 24:1001-1010.

20. Handleya S, Benton WC (2013) The influence of task and locationspecific complexity on the control and coordination costs in global outsourcing relationships. J of Operations Management 31:10-128.

21. Whitten D, Chakrabarty S, Wakefield R (2010) The strategic choice to continue outsourcing, switch vendors or backsource: Do switching costs matter? Information and Management 47:167-175.

22. Valazza M, Wada G (2001) Creating a successful partnership with a contract manufacturer. Pharm Technol Europe 13: 26-34.

23. Fisher C (2003) Inside the outsourcing relationship-real life lessons from contract manufacturing services partners. Bio-Pharm 16.

24. Catalano T (2013) Essential Elements for a GMP Analytical Chemistry Department. Springer Science and Business Media New York. 